\title{
Complications and outcomes of vasopressor usage in acute traumatic central cord syndrome
}

\author{
William J. Readdy, BS, ${ }^{1}$ William D. Whetstone, MD, ${ }^{1,2}$ Adam R. Ferguson, $\mathrm{PhD},{ }^{1}$ \\ Jason F. Talbott, MD, PhD, ${ }^{1,3}$ Tomoo Inoue, MD, PhD, ${ }^{1}$ Rajiv Saigal, MD, PhD, ${ }^{1}$ \\ Jacqueline C. Bresnahan, PhD, ${ }^{1}$ Michael S. Beattie, PhD, ${ }^{1}$ Jonathan Z. Pan, MD, PhD, ${ }^{1,4}$ \\ Geoffrey T. Manley, MD, PhD, ${ }^{1}$ and Sanjay S. Dhall, MD' \\ 1Department of Neurological Surgery, Brain and Spinal Injury Center; and Departments of ${ }^{2}$ Emergency Medicine, \\ ${ }^{3}$ Radiology and Biomedical Imaging, and ${ }^{4}$ Anesthesia, University of California, San Francisco, California
}

\begin{abstract}
OBJECT The optimal mean arterial pressure (MAP) for spinal cord perfusion after trauma remains unclear. Although there are published data on MAP goals after spinal cord injury (SCI), the specific blood pressure management for acute traumatic central cord syndrome (ATCCS) and the implications of these interventions have yet to be elucidated. Additionally, the complications of specific vasopressors have not been fully explored in this injury condition.

METHODS The present study is a retrospective cohort analysis of 34 patients with ATCCS who received any vasopressor to maintain blood pressure above predetermined MAP goals at a single Level 1 trauma center. The collected variables were American Spinal Injury Association (ASIA) grades at admission and discharge, administered vasopressor and associated complications, other interventions and complications, and timing of surgery. The relationship between the 2 most common vasopressors-dopamine and phenylephrine-and complications within the cohort as a whole were explored, and again after stratification by age.
\end{abstract}

RESULTS The mean age of the ATCCS patients was 62 years. Dopamine was the most commonly used primary vasopressor ( $91 \%$ of patients), followed by phenylephrine (65\%). Vasopressors were administered to maintain MAP goals for a mean of 101 hours. Neurological status improved by a median of 1 ASIA grade in all patients, regardless of the choice of vasopressor. Sixty-four percent of surgical patients underwent decompression within 24 hours. There was no observed relationship between the timing of surgical intervention and the complication rate. Cardiogenic complications associated with vasopressor usage were notable in $68 \%$ of patients who received dopamine and $46 \%$ of patients who received phenylephrine. These differences were not statistically significant (OR with dopamine 2.50 [95\% $\mathrm{Cl} 0.82-7.78]$, $p=0.105)$. However, in the subgroup of patients $>55$ years, dopamine produced statistically significant increases in the complication rates when compared with phenylephrine (83\% vs 50\% for dopamine and phenylephrine, respectively; OR with dopamine 5.0 [95\% Cl 0.99-25.34], $p=0.044$ ).

CONCLUSIONS Vasopressor usage in ATCCS patients is associated with complication rates that are similar to the reported literature for SCI. Dopamine was associated with a higher risk of complications in patients $>55$ years. Given the increased incidence of ATCCS in older populations, determination of MAP goals and vasopressor administration should be carefully considered in these patients. While a randomized control trial on this topic may not be practical, a multiinstitutional prospective study for SCI that includes ATCCS patients as a subpopulation would be useful for examining MAP goals in this population.

http://thejns.org/doi/abs/10.3171/2015.2.SPINE14746

KEY WORDS ATCCS; central cord; traumatic spinal cord injury; cervical spine; spinal cord perfusion; vasopressors; dopamine; trauma

ABBREVIATIONS AANS/CNS = American Association of Neurological Surgeons and Congress of Neurological Surgeons; ASIA = American Spinal Injury Association; ATCCS = acute traumatic central cord syndrome; ICU = intensive care unit; ISP = intraspinal pressure; $\mathrm{MAP}=$ mean arterial pressure; SCI = spinal cord injury; SCPP = spinal cord perfusion pressure.

SUBMITTED July 29, 2014. ACCEPTED February 5, 2015.

INCLUDE WHEN CITING Published online July 31, 2015; DOI: 10.3171/2015.2.SPINE14746.

DISCLOSURE Dr. Dhall reports receiving speaking honoraria from DePuy and Globus. 
$\mathrm{S}$ INCE Schneider colleagues' well-known description of acute traumatic central cord syndrome (ATCCS) in 1954, significant research has focused on the management of these cases..$^{18}$ In recent years, there has been an increased focus on ATCCS, as this represents the most common form of incomplete spinal cord injury (SCI). ${ }^{3}$ Additionally, ATCCS complications have been shown to increase in elderly patients. ${ }^{14}$ As the US population ages, expanding knowledge of ATCCS will only become more important. Given the potentially debilitating nature of these injuries, and their impact on our society, it is important to explore the medical and surgical management of ATCCS. ${ }^{5}$

Many recent studies on SCI and ATCCS have focused on the timing of surgical intervention and decompression and report mixed results, all citing the need for additional prospective studies. ${ }^{19,24,26}$ These studies, along with recent prospective investigations, suggest that early surgical intervention (decompression within 24 hours of SCI) may improve long-term prognosis. ${ }^{2,9,24}$ While the focus on surgical decompression, efficacy, and timing is an important aspect of ATCCS management, little focus has been placed on medical management and perfusion for these patients. ${ }^{2}$

As part of the medical management of SCI, there has been an increased focus on vasopressor utilization. Previous studies have linked vasopressor support to improved outcomes, but recognized that there are no validated protocols for the implementation of these interventions. ${ }^{15,21}$ The 2013 American Association of Neurological Surgeons and Congress of Neurological Surgeons (AANS/CNS) guidelines for cervical SCI treatment recommended raising the mean arterial blood pressure of acute SCI patients to the range of $85-90 \mathrm{~mm} \mathrm{Hg}$, while acknowledging that further research should be conducted to formulate consistent guidelines and protocols. ${ }^{2,17}$ As this recommendation was made primarily based on a single, large, retrospective study with significant positive results, the AANS/CNS author group encouraged more robust research as it relates to the medical management of cervical SCI subpopulations.

Concurrently with ongoing research investigating vasopressor utilization for traumatic SCI, poor clinical outcomes have been reported in the setting of early vasopressor use for critically injured, nonneurosurgical, trauma patients. ${ }^{16}$ Excluding traumatic brain injury and SCI, Plurad et al. found a significant, fluid status-independent association between early vasopressor administration and mortality. ${ }^{16}$ In the setting of septic shock and cardiogenic shock, other studies found that dopamine was associated with significantly higher complication rates and mortality when compared with norepinephrine. ${ }^{6,7}$ To the best of our knowledge, no previous studies have evaluated the role and potential risks of vasopressor utilization specifically in ATCCS patients. Due to the frequency of ATCCS, and its increased incidence in the aging population, research related to the medical management of these patients has become increasingly important, particularly in light of the current lack of universal standards. ${ }^{2,3,14,17}$ In this study, we explored the ATCCS subpopulation of acute SCI in order to establish a better understanding of perfusion pressure management in an effort to complement ongoing research related to surgical timing and decompression, and to provide a detailed analysis of complications in these injuries. ${ }^{9}$ Additionally, we hypothesized that specific vasopressors may be linked to higher complication rates, along the lines of recent research on critical trauma and shock. ${ }^{67,12}$

\section{Methods}

This study was reviewed and approved by the Committee on Human Research at the University of California, San Francisco, with an exemption from individual patient consent. We performed this retrospective cohort study of patients at a single Level 1 trauma center and created a database for analysis in REDCap (Research Electronic Data Capture), which was hosted at the University of California, San Francisco, in order to maintain data security and validity.

\section{Population Selection}

Potential study participants were identified by querying a preexisting database maintained by the Department of Neurological Surgery, which included all sequential patients with a principal diagnosis of SCI (ICD code: 953-957) from 2005-2011. This database includes 131 patients who met the following criteria: 1 ) age $\geq 18$ years; 2) presence of SCI; 3) admission to the intensive care unit (ICU); and 4) received vasopressors to meet mean arterial pressure (MAP) goals for greater than 24 hours. For this study, we had the specific additional inclusion criteria of the presence of central cord syndrome, as defined by the 2013 AANS/CNS guidelines for the management of ATCCS. ${ }^{2}$ From this subpopulation, an additional comprehensive chart review was conducted to elucidate a better understanding of the injury and its management.

\section{Population Characteristics, Complications, and Outcomes}

The following variables were collected from the Department of Neurological Surgery database: sex, age, year of injury, vasopressor administration (type and duration of administration), American Spinal Injury Association (ASIA) grade on admission and discharge, level of SCI, and characteristics of injury. These data were then expanded by a blinded researcher by adding variables, including trauma characteristics, administration of methylprednisolone or other steroids, hospital length of stay, ICU length of stay, and surgical interventions. These data were collected from all aspects of the chart, including discharge summaries, nursing notes, progress notes, consent for procedures, operative reports, rehabilitation notes, and pharmacy records. The blinded researcher also independently verified the original data obtained from the departmental database.

Another researcher also reviewed the complications. These included surgical infections, wound complications, hospital-acquired infections, respiratory failure, hemodynamic complications, and cardiogenic complications. Cardiogenic complications included elevated troponins, atrial fibrillation, ventricular tachycardia, significant tachycardia (heart rate $>130 \mathrm{bpm}$ ), and significant bradycardia (heart rate $<50 \mathrm{bpm}$ ). Additionally, invasive procedures-including intubation, tracheostomies, gastrostomies, arterial 
line placement, central line placement, and peripherally inserted central catheters - were reviewed as indicative of advanced medical care. Outcomes were determined based on improvement in neurological function, as indicated by the ASIA grade from admission to discharge and/or death. ASIA grade was selected as the measure of neurological function, given the recommendations of the AANS/CNS guidelines for the classification of cervical injuries and significant validation for the prognostic value of the ASIA grade..$^{10,13,25}$

\section{Statistical Analysis}

Descriptive statistics were used to examine the complications associated with vasopressor administration in ATCCS patients. All statistical analyses were performed using SPSS statistical analysis software (IBM SPSS Statistics for Macintosh, version 22.0). For all univariate analyses, the continuous variables are presented as the means with corresponding standard deviations. The univariate descriptions of the categorical data are presented as the incidence and associated percentages. Complications associated with the administration of the 2 primary vasopressors-dopamine and phenylephrine-were compared utilizing the chi-square and Fisher exact tests. Given the high incidence of ATCCS in older patients, an additional subanalysis was performed between patients older and younger than 55 years, which is an age cutoff point based on the recent literature on vasopressors. ${ }^{16}$ These groups were further compared using the Pearson chi-square test for dichotomous variables and 2-tailed t-tests for continuous data. For all statistical comparisons, statistical significance was defined as $\mathrm{p} \leq 0.05$. The odds ratios were calculated for all cross-tabulated descriptive statistics with accompanying $95 \%$ confidence intervals.

\section{Results \\ Cohort Description and Management}

Of the 131 patients in the original database, 34 were determined to have ATCCS, as defined by the inclusion criteria, with complete records available for analysis. As shown in Table 1, $28(82 \%)$ were male and $6(18 \%)$ were female with a mean age of $61.53 \pm 16.33$ years. The average hospital length of stay was $18.64 \pm 19.09$ days with an average of $11.67 \pm 13.73$ days of care in the ICU. The acute SCI methylprednisolone protocol was administered to 20 patients $(59 \%)$, while 14 patients $(41 \%)$ were determined to be ineligible for the steroid protocol based on the decisions of their managing surgeon. Chart review indicated that methylprednisolone was not administered for multiple reasons, including medical comorbidities, injury severity, surgeon preference, and timing outside of the initial window of therapeutic intervention. Patients who did not receive steroids presented with more severe injury when compared with the group that received steroids, as indicated by higher average Injury Severity Scores (28 vs 21 , respectively), but this did not reach significance $(\mathrm{p}=$ 0.353 ). There was no statistical difference in cardiogenic complications between patients who received or did not receive steroid protocols $(85.0 \%$ for patients who received steroids [17 of 20] vs $64.29 \%$ for patients who did not re-
TABLE 1. Descriptive demographics*

\begin{tabular}{lc}
\hline \multicolumn{1}{c}{ Variable } & Value \\
\hline No. of patients & 34 \\
\hline Male & $28(82.35)$ \\
\hline Female & $6(17.65)$ \\
\hline Mean age (yrs) & $61.53 \pm 16.33$ \\
\hline Mean MAP goals >85 mm Hg (hrs) & $100.78 \pm 47.54$ \\
\hline Mean ISS & $23.52 \pm 17.91$ \\
\hline Steroids administered & $20(58.82)$ \\
\hline No steroids & $14(41.18)$ \\
\hline Surgery in <24 hrs & $16(47.06)$ \\
\hline Surgery in >24 hrs & $9(26.47)$ \\
\hline No surgery & $9(26.47)$ \\
\hline ASIA grade improvement & $19(55.88)$ \\
\hline No ASIA grade improvement & $15(44.12)$ \\
\hline Mean ICU LOS (days) & $11.67 \pm 13.73$ \\
\hline Mean hospital LOS (days) & $18.64 \pm 19.09$ \\
\hline Mortality & $2(5.88)$ \\
\hline
\end{tabular}

ISS = Injury Severity Score; LOS = length of stay.

* Continuous variables are reported as the mean $\pm \mathrm{SD}$; categorical variables are reported as number (\%).

ceive steroids [ 9 of 14]; OR with steroids 3.142 [95\% CI 0.608-16.289], $\mathrm{p}=0.161$ ). Additionally, when comparing the steroid group to the nonsteroid group, there were no statistical differences in any of the measured complication rates or outcomes. Decompressive surgery was performed in the first 24 hours in 16 patients (47\%). Surgical intervention after 24 hours was noted in an additional 9 patients $(26 \%)$, with the remaining 9 patients having no surgical intervention. Those patients who did not have surgical intervention either elected against the procedure, were medically unstable to the extent that the risks outweighed the benefits, or saw improvement without decompression. Of the patients who underwent decompressive surgery, $64 \%$ (16 of 25 patients) underwent surgery within the first 24 hours.

\section{Neurological Outcomes}

Table 2 provides a detailed review of the cohort stratified by ASIA grade on admission. At the time of admission, there were 8 ASIA Grade A (24\%), 5 Grade B (14\%), 8 Grade C (24\%), 12 Grade D (35\%), and 1 Grade E (3\%) patients. Improvement of at least 1 ASIA grade was observed in 19 patients (56\%); the remaining 15 patients had the same ASIA grade at admission and discharge. Two patients died during the course of their treatment, resulting in a mortality rate of $6 \%$. One patient suffered from pulseless electrical activity in the field and was resuscitated, but never recovered from other injuries. He was treated aggressively but his Glasgow Coma Scale score never improved above $5 \mathrm{~T}$, and the family elected to withdraw care in the context of multiple organ failure. The second death occurred in an elderly patient who fell while standing. The patient developed significant multisystem organ failure that required mechanical ventilation and acute renal replacement therapy. The patient also required reversal of 
TABLE 2. Incidence of results stratified by initial ASIA grade*

\begin{tabular}{|c|c|c|c|c|c|}
\hline \multirow[b]{2}{*}{ Variable } & \multicolumn{5}{|c|}{ ASIA Grade } \\
\hline & $A(n=8)$ & $B(n=5)$ & $C(n=8)$ & $D(n=12)$ & $E(n=1)$ \\
\hline 1-grade improvement & $0(0)$ & $2(40)$ & $6(75)$ & $5(41.67)$ & $0(0)$ \\
\hline 2-grade improvement & $2(25)$ & $2(40)$ & $1(12.5)$ & $0(0)$ & $0(0)$ \\
\hline 3-grade improvement & $1(12.5)$ & $0(0)$ & $0(0)$ & $0(0)$ & $0(0)$ \\
\hline No improvement & $5(62.5)$ & $1(20)$ & $1(12.5)$ & $7(58.33)$ & $1(100)$ \\
\hline Dopamine administered & $8(100)$ & $5(100)$ & $6(75)$ & $11(91.67)$ & $1(100)$ \\
\hline Phenylephrine administered & $5(62.5)$ & $4(80)$ & $4(50)$ & $9(75)$ & $0(0)$ \\
\hline Dopamine administered first & $6(75)$ & $4(80)$ & $6(75)$ & $10(83.33)$ & $1(100)$ \\
\hline Phenylephrine administered first & $2(25)$ & $1(20)$ & $2(25)$ & $2(16.67)$ & $1(100)$ \\
\hline Dopamine complications & $5 / 8(62.5)$ & $4 / 5(80)$ & $4 / 6(66.67)$ & $7 / 11(63.64)$ & $1 / 1(100)$ \\
\hline Phenylephrine complications & $4 / 5(80)$ & $2 / 4(50)$ & $2 / 4(50)$ & $2 / 9(22.22)$ & $0 / 0(0)$ \\
\hline Pneumonia & $3(37.5)$ & $2(40)$ & $1(12.5)$ & $0(0)$ & $0(0)$ \\
\hline Respiratory failure & $8(100)$ & $4(80)$ & $1(12.5)$ & $2(16.67)$ & $0(0)$ \\
\hline Urinary tract infection & $3(37.5)$ & $2(40)$ & $2(25)$ & $1(8.33)$ & $0(0)$ \\
\hline Tracheostomy & $3(37.5)$ & $0(0)$ & $0(0)$ & $0(0)$ & $0(0)$ \\
\hline Gastrostomy & $1(12.5)$ & $0(0)$ & $0(0)$ & $1(8.33)$ & $0(0)$ \\
\hline Steroids administered & $5(62.5)$ & $1(20)$ & $5(57.5)$ & $9(75)$ & $0(0)$ \\
\hline No steroids & $3(37.5)$ & $4(80)$ & $3(37.5)$ & $325)$ & $1(100)$ \\
\hline Surgery in $<24 \mathrm{hrs}$ & $4(50)$ & $3(60)$ & $3(37.5)$ & $5(41.66)$ & $1(100)$ \\
\hline Surgery in $>24 \mathrm{hrs}$ & $3(37.5)$ & $2(40)$ & $3(37.5)$ & $2(16.66)$ & $0(0)$ \\
\hline No surgery & $1(12.5)$ & $0(0)$ & $2(25)$ & $5(41.66)$ & $0(0)$ \\
\hline Mean age (yrs) & $63.88 \pm 15.64$ & $64.40 \pm 15.19$ & $63.125 \pm 18.11$ & $60.33 \pm 15.80$ & $30 \pm 0$ \\
\hline Mean MAP goals >85 (hrs) & $103.5 \pm 43.94$ & $149.5 \pm 12.37$ & $86.29 \pm 51.93$ & $93.583 \pm 49.94$ & $72 \pm 0$ \\
\hline Average ICU LOS (days) & $26 \pm 22.25$ & $12.8 \pm 6.05$ & $6.5 \pm 5.10$ & $5.75 \pm 2.70$ & $4 \pm 0$ \\
\hline Average hospital LOS (days) & $33.88 \pm 25.83$ & $28.6 \pm 20.99$ & $12.5 \pm 10.80$ & $9.66 \pm 9.28$ & $4 \pm 0$ \\
\hline
\end{tabular}

* Continuous variables are reported as the mean $\pm \mathrm{SD}$; categorical data are reported as number (\%).

preinjury coagulopathy and suffered from multiple nosocomial infections, which ultimately resulted in his death.

\section{Vasopressor Administration}

The characteristics of vasopressor utilization can be found in Table 3. Vasopressors were administered to obtain MAP goals $>85 \mathrm{~mm} \mathrm{Hg}$ in all patients, for a mean $101 \pm 48$ hours ( 4.2 days), before being relaxed to lower goals. This mean time was affected by 2 patients who were transferred to another acute care center for management directly from the ICU while still receiving MAP goals early in their hospitalization. Eighteen patients (53\%) had their vasopressor changed due to complications, and 12 patients were concurrently administered 2 or more vasopressors (35\%). Dopamine was administered to 31 patients (91\%) for MAP

TABLE 3. Vasopressor utilization $(n=34)$

\begin{tabular}{lc}
\hline \multicolumn{1}{c}{ Administration Pattern } & No. of Patients (\%) \\
\hline Dopamine administered & $31(91.18)$ \\
\hline Phenylephrine administered & $22(64.71)$ \\
\hline Dopamine administered first & $27(79.42)$ \\
\hline Phenylephrine administered first & $7(20.59)$ \\
\hline Patients had 2 vasopressors & $18(52.94)$ \\
\hline Patients had 2 or more concurrently & $12(35.29)$ \\
\hline
\end{tabular}

goals, and phenylephrine was administered to 22 patients (65\%). A detailed delineation of the vasopressor-associated complications can be found in Table 4. For the entire cohort, there was a nonsignificant trend toward a higher complication rate with dopamine (68\% of patients who received dopamine experienced complications [21 of 31 patients] vs $45 \%$ for phenylephrine [10 of 22 patients]; OR with dopamine 2.52 [95\% CI 0.82-7.78], $\mathrm{p}=0.105$ ). In the subgroup of patients age $>55$ years, dopamine produced statistically significant increases in complications rates when compared with phenylephrine (see Table 5). This effect was not observed in a comparison of dopamine to phenylephrine in the group $<55$ years. Further analysis showed that age $>55$ years was also associated with all vasopressor complications (90\% of older patients experienced complications [18 of 20 patients] vs $52 \%$ of younger patients [8 of 14 patients]; OR for older 6.75 [95\% CI 1.1-41.00], $\mathrm{p}=0.026$ ), despite there being no significant differences in injury severity score, mean ASIA improvement, steroid administration, or length of stay, as shown in Table 6. Together these results suggest that dopamine is associated with a higher risk of complications than phenylephrine in older patients.

\section{Complications}

Twenty-nine patients experienced at least 1 complication. Table 7 summarizes the complication rates. The most 
TABLE 4. Specific complication rates by individual vasopressor

\begin{tabular}{lcc}
\hline \multirow{2}{*}{ Complication } & \multicolumn{2}{c}{ No. of Patients $(\%)^{*}$} \\
\cline { 2 - 3 } & Dopamine & Phenylephrine \\
\hline Patients w/ complications & $21(67.74)$ & $10(45.45)$ \\
\hline Patients w/ multiple complications & $2(6.45)$ & $1(4.54)$ \\
\hline Atrial fibrillation & $5(16.13)$ & $0(0)$ \\
\hline Tachycardia $(\mathrm{HR}>130 \mathrm{bpm})$ & $9(29.03)$ & $3(13.64)$ \\
\hline Bradycardia $(\mathrm{HR}<50 \mathrm{bpm})$ & $4(12.90)$ & $7(31.82)$ \\
\hline Ventricular tachycardia & $3(9.68)$ & $0(0)$ \\
\hline Troponin levels & $2(6.45)$ & $1(4.54)$ \\
\hline HR = heart rate. & \multicolumn{2}{c}{} \\
* Percentages are based on the number of patients per category.
\end{tabular}

common complications were cardiogenic complications associated with vasopressor administration that occurred in 26 patients $(76 \%)$. Four patients $(12 \%)$ experienced respiratory failure during the acute phase of their injury. An additional 10 (29\%) patients experienced respiratory failure as a complication during the course of their hospitalization. Eight patients (24\%) developed urinary tract infections, and 6 patients (18\%) developed pneumonia. Five patients $(15 \%)$ also presented with a concurrent traumatic brain injury. Additional complications and comorbidities included 1 pulmonary embolism without deep vein thrombosis, 1 pneumothorax from central line placement, 1 venous catheter infection, and 1 forehead hematoma and evacuation. No surgical site infections, deep vein thromboses, or strokes were noted.

\section{Discussion}

The reviewed cohort of ATCCS patients had cardiogenic complication rates comparable to other studies of vasopressor use in patients with SCI, although our patients received MAP goal support for less than the Level III recommendation of 7 days. ${ }^{12,17}$ Given the retrospective nature of this study, it is difficult to determine if the inability to meet MAP goals was triggered by early termination due to complications or provider discretion. Of note, the mean duration of MAP goals was determined to be approximately 4.2 days, as compared with 7 days proposed for SCI patients by Vale et al. ${ }^{21}$ While limited by the retrospective nature of this study, we believe that this shorter duration is reflective of the treating surgeon's desire to reduce the morbidity of vasopressor use, particularly after surgical decompression.

Similar to the findings in other recent studies that evaluate vasopressor-related complications for trauma and shock, dopamine was the most common first-line vasopressor administered and associated with a higher risk of complication when compared with phenylephrine., Although phenylephrine was associated with lower complication rates, it is not recommended for use in cervical injury due to its risk of inducing bradycardia. ${ }^{4}$ Despite these recommendations, we noted that almost half of the patients received treatment with phenylephrine, most commonly as a second-line treatment following complications with dopamine. Given the propensity for cardiovascular complications following SCI, including hypotensive neurogenic shock and autonomic dysreflexia-induced hypertension, optimizing vasopressor support is a critically important issue. ${ }^{20,27}$ Considering the high prevalence of ATCCS in elderly patients and our findings of increased risk of dopamine-related complications in elderly patients with ATCCS, further research is needed to determine the optimal MAP guidelines for ATCCS. ${ }^{14}$ Since ATCCS is generally a less severe injury than other forms of acute traumatic SCI, caution is warranted when determining supportive interventions, and further research is needed to elucidate the best interventions for this patient population. Our data suggest that any physician administering dopamine in the context of ATCCS, especially for patients older than 55 years, must consider the high complication rates associated with dopamine.

An understanding of vasopressor management protocols for patients with ATCCS will gain even more importance if early data on optimized spinal cord perfusion leads to improved outcomes. Werndle et al. recently reported on a prospective trial, in which intraspinal pressure (ISP) monitors were placed in patients with traumatic SCI. ${ }^{22}$ These monitors were used to observe the spinal cord perfusion pressure (SCPP) in 18 patients without any complications such as wound infections or cerebrospinal fluid leaks. Their data directly show that elevated MAP due to vasopressor augmentation does result in a direct increase in ISP and SCPP. Studies in animal models of SCI show that microvascular damage and hypoperfusion is associated with increased degeneration after SCI.8,11,23 Advanced studies with accurate monitoring via surgically implanted ISP monitors in concordance with neurological improvement scores could contribute to a better understanding of optimal MAP goals in ATCCS patients and provide clear protocols on the issue.

\section{Other Interventions}

Our patient population was treated with decompressive surgical intervention at a higher rate and with increased urgency when compared with the published rates in ATCCS and SCI. ${ }^{1,19}$ In our cohort we found that $64 \%$ of surgical patients (15 of 26 patients) underwent decompression within 24 hours. Conversely, a study examining patients from a similar time period by Aarabi et al. indicated

TABLE 5. Dopamine- vs phenylephrine-induced complications by age*

\begin{tabular}{ccccc}
\hline Cohort & Dopamine Complications & Phenylephrine Complications & OR $(95 \% \mathrm{Cl})$ & $\mathrm{p}$ Value \\
\hline Entire cohort & $21 / 31(67.74)$ & $10 / 22(45.45)$ & $2.520(0.816-7.782)$ & 0.105 \\
\hline Age $>55 \mathrm{yrs}$ & $15 / 18(83.33)$ & $7 / 14(50)$ & $5.000(0.987-25.341)$ & 0.044 \\
\hline Age $<55 \mathrm{yrs}$ & $6 / 13(46.15)$ & $3 / 8(37.5)$ & $0.700(0.116-4.232)$ & 0.697 \\
\hline * Value in boldface is statistically significant & & &
\end{tabular}


TABLE 6. Comparison of vasopressor complications by age (age $>55$ vs $<55$ years)*

\begin{tabular}{|c|c|c|c|c|}
\hline Variable & $\begin{array}{c}\text { Age }>55 \\
(n=20)\end{array}$ & $\begin{array}{c}\text { Age }<55 \\
(n=14)\end{array}$ & $\begin{array}{c}\text { OR }(95 \% \mathrm{Cl}) \\
\text { (when applicable) }\end{array}$ & $\mathrm{p}$ Value \\
\hline Mean age (yrs) & $72.55 \pm 10.875$ & $45.79 \pm 7.7073$ & & $<0.01$ \\
\hline Mean ISS & $23.73 \pm 18.642$ & $23.21 \pm 17.564$ & & 0.936 \\
\hline Mean MAP goals (hrs) & $104.83 \pm 52.922$ & $95.57 \pm 40.929$ & & 0.593 \\
\hline Mean ASIA grade improvement & $0.65 \pm 0.671$ & $0.93 \pm 0.997$ & & 0.336 \\
\hline Steroids administered & $13(65.0)$ & $7(50.0)$ & $1.857(0.461-7.482)$ & 0.382 \\
\hline ICU LOS (days) & $11.70 \pm 11.965$ & $11.64 \pm 16.402$ & & 0.991 \\
\hline Hospital LOS (days) & $16.80 \pm 15.946$ & $21.29 \pm 23.262$ & & 0.538 \\
\hline Dopamine administered & $18(90.0)$ & $13(92.9)$ & $0.692(0.057-8.470)$ & 0.773 \\
\hline Dopamine complication & $15(83.3)$ & $6(46.15)$ & $5.833(1.119-30.403)$ & 0.029 \\
\hline Phenylephrine administered & $14(70.0)$ & $8(57.1)$ & $1.750(0.420-7.288)$ & 0.44 \\
\hline Phenylephrine complication & $7(50.0)$ & $3(37.5)$ & $1.667(0.283-9.822)$ & 0.571 \\
\hline Any vasopressor complication & $18(90.0)$ & $8(57.1)$ & $6.750(1.111-41.001)$ & 0.026 \\
\hline
\end{tabular}

that $21 \%$ (9 of 42) of their patients with ATCCS underwent rapid surgical decompression within 24 hours. ${ }^{1}$ This was consistent with another retrospective study where $24 \%$ of ATCCS patients (16 of 67) who underwent decompressive surgery were treated within 24 hours. ${ }^{19}$ Ultimately, the decision to perform surgery and the timing of surgery were dependent on the treating surgeon. Given the recent results of the Surgical Timing for Traumatic Cervical Spinal Cord Injury Study (STASCIS), which indicated the benefits of early decompression, we found this difference to be significant and noteworthy. ${ }^{9}$ Steroids did not appear to have an impact on the study as the complication rates did not vary between the steroid and nonsteroid groups. The sample size of this study and the lack of significant differences in outcomes and complications between the patients who received steroids and those who did not make it difficult to draw any meaningful conclusions regarding steroids in this population.

\section{Limitations}

The primary limitations of this study were the retrospective nature and small sample size of our population. In addition, this retrospective analysis was limited to the course of acute recovery, and neurological outcomes in long-term follow-up may have provided additional insight into the effect of vasopressors. The small sample size is

TABLE 7. Complication rate $(n=34)$

\begin{tabular}{lc}
\hline \multicolumn{1}{c}{ Complication } & No. of Patients (\%) \\
\hline Pneumonia & $6(17.65)$ \\
\hline Respiratory failure on arrival & $4(11.76)$ \\
\hline Respiratory failure in hospital & $10(29.41)$ \\
\hline Urinary tract infection & $8(23.53)$ \\
\hline Tracheostomy & $3(8.82)$ \\
\hline Gastrostomy & $2(5.88)$ \\
\hline Cardiogenic & $26(76.47)$ \\
\hline Complication of any kind & $29(85.29)$ \\
\hline
\end{tabular}

reflective of the limited number of central cord injuries seen at an individual institution. This limitation may have prevented several associations from reaching significance with $p \leq 0.05$, as many associations approached this statistical cutoff. At our institution, we have adhered to protocolbased management as strictly as possible for several years. As such, nearly all of our patients with acute SCI, including ATCCS, were managed with vasopressors and MAP goals. Though we believe that this practice improves the quality of the care that we provide to patients, the downside is that this has resulted in the lack of a control group for this study. Finally, quantification of ATCCS severity was performed utilizing the ASIA grading system, and this has limitations given the asymmetrical involvement of the upper extremities associated with ATCCS.

\section{Conclusions}

Our results provide compelling data concerning vasopressor-associated complication rates in patients with central cord syndrome. We observed a complication rate of $85 \%$ for ATCCS injuries, with $76 \%$ of patients experiencing cardiogenic complications associated with vasopressor administration. As the US population continues to age, we anticipate a rise in this condition given its increased incidence in the elderly. Based on the results of our analysis, careful consideration of the risks should be made before administering dopamine in the context of ATCCS in patients over 55 years.

Establishing clear MAP guidelines for ATCCS, in addition to SCI in general, is extremely important and warrants thorough investigation. Ideally, we encourage a multicenter prospective study to elucidate the risk-benefit ratio for SCI with a subanalysis of central cord patients. Given the difficulty of establishing this type of protocol, a more rapid and financially obtainable solution may be to conduct a large, multicenter, retrospective review of SCI patients receiving vasopressors in order to compare cross-institutional outcomes and complications while also providing the statistical power to make more confident as- 
sessments of MAP goals. A subgroup analysis of central cord injuries in this type of study would also be extremely valuable for elucidating additional knowledge regarding ATCCS.

\section{References}

1. Aarabi B, Alexander M, Mirvis SE, Shanmuganathan K, Chesler D, Maulucci C, et al: Predictors of outcome in acute traumatic central cord syndrome due to spinal stenosis. J Neurosurg Spine 14:122-130, 2011

2. Aarabi B, Hadley MN, Dhall SS, Gelb DE, Hurlbert RJ, Rozzelle CJ, et al: Management of acute traumatic central cord syndrome (ATCCS). Neurosurgery 72 (Suppl 2):195-204, 2013

3. Aarabi B, Koltz M, Ibrahimi D: Hyperextension cervical spine injuries and traumatic central cord syndrome. Neurosurg Focus 25(5):E9, 2008

4. Consortium for Spinal Cord Medicine: Early acute management in adults with spinal cord injury: a clinical practice guideline for health-care professionals. J Spinal Cord Med 31:403-479, 2008

5. Dahdaleh NS, Lawton CD, El Ahmadieh TY, Nixon AT, El Tecle NE, Oh S, et al: Evidence-based management of central cord syndrome. Neurosurg Focus 35(1):E6, 2013

6. De Backer D, Aldecoa C, Njimi H, Vincent JL: Dopamine versus norepinephrine in the treatment of septic shock: a meta-analysis. Crit Care Med 40:725-730, 2012

7. De Backer D, Biston P, Devriendt J, Madl C, Chochrad D, Aldecoa C, et al: Comparison of dopamine and norepinephrine in the treatment of shock. N Engl J Med 362:779-789, 2010

8. Fassbender JM, Whittemore SR, Hagg T: Targeting microvasculature for neuroprotection after SCI. Neurotherapeutics 8:240-251, 2011

9. Fehlings MG, Vaccaro A, Wilson JR, Singh A, W Cadotte D, Harrop JS, et al: Early versus delayed decompression for traumatic cervical spinal cord injury: results of the Surgical Timing in Acute Spinal Cord Injury Study (STASCIS). PLoS ONE 7:e32037, 2012

10. Hadley MN, Walters BC, Aarabi B, Dhall SS, Gelb DE, Hurlbert RJ, et al: Clinical assessment following acute cervical spinal cord injury. Neurosurgery 72 (Suppl 2):40-53, 2013

11. Han S, Arnold SA, Sithu SD, Mahoney ET, Geralds JT, Tran $\mathrm{P}$, et al: Rescuing vasculature with intravenous angiopoietin-1 and alpha $v$ beta 3 integrin peptide is protective after spinal cord injury. Brain 133:1026-1042, 2010

12. Inoue T, Manley GT, Patel N, Whetstone WD: Medical and surgical management after spinal cord injury: vasopressor usage, early surgerys, and complications. J Neurotrauma 31:284-291, 2014

13. Kirshblum SC, Memmo P, Kim N, Campagnolo D, Millis S: Comparison of the revised 2000 American Spinal Injury Association classification standards with the 1996 guidelines. Am J Phys Med Rehabil 81:502-505, 2002

14. Newey ML, Sen PK, Fraser RD: The long-term outcome after central cord syndrome: a study of the natural history. J Bone Joint Surg Br 82:851-855, 2000

15. Ploumis A, Yadlapalli N, Fehlings MG, Kwon BK, Vaccaro AR: A systematic review of the evidence supporting a role for vasopressor support in acute SCI. Spinal Cord 48:356362, 2010

16. Plurad DS, Talving P, Lam L, Inaba K, Green D, Demetriades D: Early vasopressor use in critical injury is associated with mortality independent from volume status. J Trauma 71:565-572, 2011

17. Ryken TC, Hurlbert RJ, Hadley MN, Aarabi B, Dhall SS, Gelb DE, et al: The acute cardiopulmonary management of patients with cervical spinal cord injuries. Neurosurgery 72 (Suppl 2):84-92, 2013
18. Schneider RC, Cherry G, Pantek H: The syndrome of acute central cervical spinal cord injury; with special reference to the mechanisms involved in hyperextension injuries of cervical spine. J Neurosurg 11:546-577, 1954

19. Stevens EA, Marsh R, Wilson JA, Sweasey TA, Branch CL Jr, Powers AK: A review of surgical intervention in the setting of traumatic central cord syndrome. Spine J 10:874880,2010

20. Summers RL, Baker SD, Sterling SA, Porter JM, Jones AE: Characterization of the spectrum of hemodynamic profiles in trauma patients with acute neurogenic shock. J Crit Care 28:531.e1-531.e5, 2013

21. Vale FL, Burns J, Jackson AB, Hadley MN: Combined medical and surgical treatment after acute spinal cord injury: results of a prospective pilot study to assess the merits of aggressive medical resuscitation and blood pressure management. J Neurosurg 87:239-246, 1997

22. Werndle MC, Saadoun S, Phang I, Czosnyka M, Varsos GV, Czosnyka ZH, et al: Monitoring of spinal cord perfusion pressure in acute spinal cord injury: initial findings of the injured spinal cord pressure evaluation study. Crit Care Med 42:646-655, 2014

23. Whetstone WD, Hsu JY, Eisenberg M, Werb Z, Noble-Haeusslein LJ: Blood-spinal cord barrier after spinal cord injury: relation to revascularization and wound healing. J Neurosci Res 74:227-239, 2003

24. Wilson JR, Arnold PM, Singh A, Kalsi-Ryan S, Fehlings MG: Clinical prediction model for acute inpatient complications after traumatic cervical spinal cord injury: a subanalysis from the Surgical Timing in Acute Spinal Cord Injury Study. J Neurosurg Spine 17 (1 Suppl):46-51, 2012

25. Wilson JR, Grossman RG, Frankowski RF, Kiss A, Davis AM, Kulkarni AV, et al: A clinical prediction model for longterm functional outcome after traumatic spinal cord injury based on acute clinical and imaging factors. J Neurotrauma 29:2263-2271, 2012

26. Yoshihara H, Yoneoka D: Trends in the treatment for traumatic central cord syndrome without bone injury in the United States from 2000 to 2009. J Trauma Acute Care Surg 75:453-458, 2013

27. Zhang Y, Guan Z, Reader B, Shawler T, Mandrekar-Colucci S, Huang K, et al: Autonomic dysreflexia causes chronic immune suppression after spinal cord injury. J Neurosci 33:12970-12981, 2013

\section{Author Contributions}

Conception and design: all authors. Acquisition of data: Readdy, Whetstone, Inoue. Analysis and interpretation of data: Dhall, Readdy, Whetstone. Drafting the article: Dhall, Readdy, Talbott. Critically revising the article: Dhall, Readdy, Whetstone, Ferguson, Talbott, Saigal, Bresnahan, Pan, Manley. Reviewed submitted version of manuscript: all authors. Approved the final version of the manuscript on behalf of all authors: Dhall. Statistical analysis: Readdy, Ferguson, Beattie. Study supervision: Dhall, Manley.

\section{Supplemental Information \\ Previous Presentation}

Portions of this work were accepted and presented as an oral presentation by Dr. Sanjay Dhall to the Annual Meeting of the Congress of Neurological Surgeons, Boston, Massachusetts, October $18-22,2014$.

\section{Correspondence}

Sanjay Dhall, Department of Neurological Surgery, University of California, San Francisco, 505 Parnassus Ave., Box 0112, San Francisco, CA 94143. email: sanjaydhall@ gmail.com. 